\title{
Comparative analysis of ACSR and ACCC conductors on corona effect for lightning surge studies
}

\begin{abstract}
Corona effect plays an important role in determining the distortion and attenuation of the surges travelling along overhead transmission lines and thus could possibly cause the backflashover. This this paper presents a comparative analysis between the two conductors used for $275 \mathrm{kV}$ transmission line in term of corona effect when subjected to the lightning surge propagation studies. The two conductors are the Aluminium Conductor Steel Reinforced (ACSR) and the new High-Temperature Low Sag (HTLS) conductor, known as Aluminium Conductor Composite Core (ACCC). Simulation using section by section method for a single conductor were simulated using PSCAD/EMTDC software. The analyses were done by comparing the results with three parameters that known to be affected the corona, namely the different conductor radius, the heights of the conductor above ground level, and the segment length used for a single conductor. The finding that can be drawn from the study is that the presence of ACCC conductor are found to be more efficient in reducing the corona effect than the ACSR conductors apart from other advantages such as higher current capacity and lesser losses as compared to ACSR.
\end{abstract}

Keyword: Conductor; ACCC; Corona; Transmission line; Lightning 